\title{
Background suppression in the JUNO experiment
}

\author{
Yury Gornushkin ${ }^{1}$ \\ Joint Institute for Nuclear Research, 6, Joliot-Curie str., Dubna, 141980, Russia \\ E-mail: gornushkeyandex.ru
}

\section{Vit Vorobel}

Faculty of Math and Physics, Charles University, Prague 12116 Praha 2, Ke Karlovu 3, Czech Republic

E-mail: vit.vorobelemff.cuni.cz

\section{on behalf of the JUNO Collaboration}

The JUNO (Jiangmen Underground Neutrino Observatory) experiment is under preparation in China by the international collaboration. The main goal of the project is to determine the neutrino mass hierarchy by precise measurement of the energy spectrum of the antineutrinos from the nuclear reactors with the help of the $20 \mathrm{kt}$ liquid scintillator underground detector located at a distance of $53 \mathrm{~km}$. Data taking should begin in 2020. The performance of the JUNO experiment is related to its capability to suppress or at least to control the background processes, which may have the same signature as the antineutrino signal. There are several sources of the background but the most dangerous one is due to the cosmogenic isotopes produced in nuclear spallation processes in cosmic muons interactions. The Veto system of the JUNO detector is used for muon detection, muon induced background study and its reduction. It consists of the Top Tracker system and the Water Cherenkov detector. The Water Cherenkov detector is a pool filled with purified water and instrumented with PMTs surrounding the central antineutrino detector. The Top Tracker, made of scintillating strips, covers $>1 / 3$ of the top area with 3 layers and helps to reconstruct the cosmic muons direction. This independent muon information will help muon tagging, track reconstruction and efficiency study to understand and reduce cosmogenic backgrounds. Effect of cosmic muons, cosmogenic isotopes, spallation neutrons, and natural radioactivity as well as the design of the Veto system detectors, their performances and the strategy for the background reduction are presented.

38th International Conference on High Energy Physics

3-10 August 2016

\section{${ }^{1}$ Speaker}


Chicago, USA

\section{Introduction}

The main goal of JUNO is to determine the neutrino mass hierarchy, which is one of the most important unsolved problems related to neutrinos [1]. The reactor antineutrinos in JUNO are detected via the inverse beta decay (IBD) by measuring the correlated positron and neutron signals. The detector design [2] allows to measure the neutrino spectrum precisely, and through observation of the distortion of the spectrum the sign of neutrino mass ordering can be determined. Compared to the small number of signal events (60/day), the number of background events is high due to the large volume of detector (20 kton). To suppress the backgrounds, all the materials and components are a subject of strong radioactive control. Fiducial volume of the Central Detector (CD) is protected by the surrounding waterpool filled with purified water. Cut selections on the event time and space correlation will strongly suppress the accidental background. The major concern is the cosmic muon induced backgrounds, because they are hard to remove. They are mainly ${ }^{9} \mathrm{Li} /{ }^{8} \mathrm{He}$ isotopes from muon spallation and muon shower particles, and fast neutron background in the detector from muon induced high energy neutrons.

\section{JUNO Veto detectors.}

In order to reduce the backgrounds, the detector will be placed underground with about $700 \mathrm{~m}$ rock on top of the experimental hall, and a veto system will tag muons to suppress the events related to them. The muon rate is estimated at about $0.003 \mathrm{~Hz} / \mathrm{m}^{2}$ with the average energy of about $214 \mathrm{GeV}$. The high energy cosmic muons produce a large number of neutrons in the rocks and other materials surrounding the CD those can produce a background in the $\mathrm{CD}$ which mimics the inverse beta decay signal. In order to protect the $\mathrm{CD}$ from the neutrons and natural radioactivity in the surrounding rocks, it will be surrounded by the Water Cherenkov (WC) detector with 20-30 kt of ultrapure water providing at least $3.2 \mathrm{~m}$ of water shield (Fig.1). Circulation water system (one volume circulation per $\sim 2$ week) keeps a good water quality including radon control $(<0.2 \mathrm{~Bq} / \mathrm{m} 3)$. Tyvek reflecting film coats the surface to increase light collection efficiency. Compensation coils system will shield the earth magnetic field to keep the PMT performance. About 2000 of 20 inch PMTs placed on the sphere and the wall of the WC will provide registration of the Cherenkov light of cosmic muons, thus reducing cosmogenic background related to them. The muon detection efficiency with water pool only is expected to be $>95 \%$, though with a limited spatial resolution $(\sim 1 \mathrm{~m})$. The most important cosmogenic background of ${ }^{9} \mathrm{Li} /{ }^{8} \mathrm{He}$ isotopes results from the muons and the 
shower particles spallation in the scintillator. The beta-n decay of ${ }^{9} \mathrm{Li} /{ }^{8} \mathrm{He}$ would mimic the inverse beta decay events. The number of ${ }^{9} \mathrm{Li} /{ }^{8} \mathrm{He}$ background events is estimated to be $\sim 80$ /day. Without proper muon veto, their existence would greatly reduce JUNO's capability to determine the neutrino mass hierarchy. The ${ }^{9} \mathrm{Li} /{ }^{8} \mathrm{He}$ background will be reduced by excluding a certain cylindrical region $(\sim 3 \mathrm{~m}$ radius $)$ along the muon track within a certain period of time $(\sim 1.2 \mathrm{~s})$ after the muon had passed through the detector. Therefore, the background reduction depends on precise muon track reconstruction. The Top Tracker (TT) detector on top of the WC (Fig.1) will help with muon tagging as well as track reconstruction independently of the CD and WC. With present design, TT will have 3 layers of about $20 \times 47 \mathrm{~m}^{2}$ made of plastic scintillator strips $(26 \mathrm{~mm}$ wide) providing $\mathrm{X}-\mathrm{Y}$ coordinates of muons with $\sim 1 \mathrm{~cm}$ accuracy. The tracking results from the TT can be extrapolated to the central detector providing spatial resolution of $20 \mathrm{~cm}$ at the bottom of CD. Therefore, TT can provide an independent measurement of the ${ }^{9} \mathrm{Li} /{ }^{8} \mathrm{He}$ background events distribution along the muon track, which can improve the accuracy of the residual background estimation after applying the veto zone. Tab.1 and Fig. 2 present the expected contribution of main sources of the background events and the scheme of their reduction down to acceptable level.
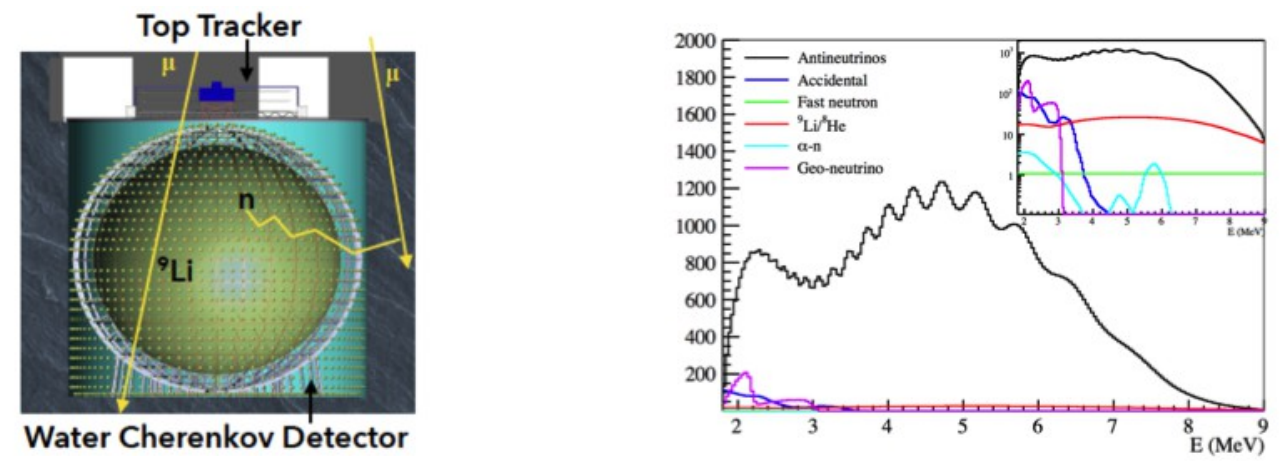

Figure 1. Schematic view of the JUNO veto detectors Figure 2. Contribution of the main backgrounds

Table 1. The main backgrounds and their reduction in JUNO.

\begin{tabular}{|c|c|c|c|c|c|c|c|}
\hline Selection & IBD efficiency & IBD & $\mathrm{Geo}-\nu \mathrm{s}$ & Accidental & ${ }^{9} \mathrm{Li} /{ }^{8} \mathrm{He}$ & Fast $n$ & $(\alpha, n)$ \\
\hline- & - & 83 & 1.5 & $\sim 5.7 \times 10^{4}$ & 84 & - & - \\
\hline Fiducial volume & $91.8 \%$ & 76 & 1.4 & \multirow{3}{*}{410} & 77 & \multirow[t]{5}{*}{0.1} & \multirow[t]{5}{*}{0.05} \\
\hline Energy cut & $97.8 \%$ & \multirow{3}{*}{73} & \multirow{3}{*}{1.3} & & \multirow{3}{*}{71} & & \\
\hline Time cut & $99.1 \%$ & & & & & & \\
\hline Vertex cut & $98.7 \%$ & & & 1.1 & & & \\
\hline Muon veto & $83 \%$ & 60 & 1.1 & 0.9 & 1.6 & & \\
\hline Combined & $73 \%$ & 60 & \multicolumn{5}{|c|}{3.8} \\
\hline
\end{tabular}




\section{Summary}

The JUNO detector design is being optimized. At present approach, the main backgrounds, including the contribution of cosmogenic isotopes, are estimated to be acceptable. Further work on the background suppression is ongoing with a hope to reduce it even more before the detector assembly in 2019 and start of data taking in 2020.

\section{References}

[1] Fengpeng An et al., Neutrino physics with JUNO, J.Phys.G43 (2016) 3030401 [physics . ins$\operatorname{det} / 1507.05613]$

[2] Z.Djurcic et al., JUNO Conceptual Design report, [physics.ins-det/1508.07166] 